\title{
Mass deposition and fluid flow in stenotic arteries: Rectangular and half-circular models
}

\author{
Dipak Kumar Mandal ${ }^{1}$, Somnath Chakrabarti ${ }^{2}$ \\ ${ }^{1}$ Department of Mechanical Engineering, College of Engineering and Management, Kolaghat, India \\ ${ }^{2}$ Department of Mechanical Engineering, Bengal Engineering and Science University, Shibpur, India \\ Email:dipkuma@yahoo.com, somnathbec@rediffmail.com
}

Received 7 September 2013; revised 15 October 2013; accepted 29 October 2013

Copyright (C) 2013 Dipak Kumar Mandal, Somnath Chakrabarti. This is an open access article distributed under the Creative Commons Attribution License, which permits unrestricted use, distribution, and reproduction in any medium, provided the original work is properly cited. In accordance of the Creative Commons Attribution License all Copyrights (C) 2013 are reserved for SCIRP and the owner of the intellectual property Dipak Kumar Mandal, Somnath Chakrabarti. All Copyright (C) 2013 are guarded by law and by SCIRP as a guardian.

\section{ABSTRACT}

Mass deposition inside the artery wall may play a significant role in the development of the disease atherosclerosis. Locally elevated concentrations of LDL in the arterial wall are considered to be the initiator of atherosclerotic plaque formation. In this study, an attempt has been made to study initially the effect of fluid dynamic parameters on the disease and finally proposed a concept, from the idea of basic flow characteristics in constricted arteries, for the assessment of mass deposition in the arterial wall to some extent for rectangular as well as half circular stenosed models. Reynolds numbers are chosen as $\mathbf{1 0 0}$, 200, 300 and 400 and percentage of restrictions as $30 \%, 50 \%, 70 \%$ and $90 \%$ respectively. The governing Navier-Stokes and continuity equations are solved in the artery lumen with the commercial CFD code ANSYS 12.1. The pressure-velocity coupling equations are solved by SIMPLE (Semi-Implicit Method for Pressure-Linked Equations) algorithm. The studies on pressure drop at stenosis zone and flow separation zone reveal that the effect of percentage of restriction is more dominant than Reynolds number on the progression of the disease, atherosclerosis for any shaped restriction. The mass deposition results of rectangular and half circular stenotic models motivate to conclude that the effect of percentage of restriction is more prone to the disease than that of Reynolds number. Half circular stenotic shape insists for the less chance of mass deposition in the arterial wall compared to rectangular shaped restriction.

Keywords: Atherosclerosis; Deposition; Pressure Drop; Velocity Contour

\section{INTRODUCTION}

Atherosclerosis is a disease of the coronary, carotid, and other proximal arteries that involve a distinctive accumulation of large molecules such as low-density lipoprotein (LDL) and other lipid-bearing materials due to mass transport in the arterial wall. This deposition of low density lipoprotein (LDL) has received considerable interest in the recent years due to its significant work in the early stages of atherosclerosis. It is a progressive disease characterized by localized plaques that form within the artery wall. As the disease progresses, these plaques enlarge and, either directly or indirectly, lead to impairment of blood flow in the concerned arterial system or artery. This in turn can have serious consequences, such as blockage of the coronary arteries (leading to myocardial infarction), femoral arteries and carotid arteries (leading to strokes as plaques detach and occlude the cerebral vasculature). Fluid dynamics of blood (hemodynamics) is considered to play a major role in the initiation and progression of atherosclerosis. The hemodynamic behaviour of the blood flow in arterial stenoses bears some important aspects due to engineering interest as well as feasible medical applications.

Stangeby and Ethier [1] have reported the fluid flow and mass transfer of LDL in a stenotic artery. The results show an elevated LDL concentration at the downstream side of the stenosis. The mass transport on symmetric and non-symmetric stenotic artery models was studied by Kaazempur-Mofrad et al. [2]. The complex flow field due to the non-symmetric stenosis affects the mass transfer patterns substantially different from those exhibited by the symmetric stenosis. Chakravarty and Sen [3] have performed the study of the blood flow and convectiondominated diffusion processes in a model bifurcated ar- 
tery under stenotic conditions. In their work, they have studied the effects of constricted flow characteristics and the wall motion on the wall shear stress, the concentration profile and the mass transfer. The influences of WSS on LDL transport by modeling the blood flow and solute transport in the lumen and arterial wall are investigated by Nanfeng Sun et al. [4]. The unsteady non-Newtonian blood flow and mass transfer in symmetric and nonsymmetric stenotic arteries are numerically simulated by Valencia and Villanueva [5], where they have considered the fluid-structure interaction in their simulation. Valeria et al. [6] have made a simple model for the plaque growth rate based on the accumulation of oxidized LDL in the artery wall, including a dependence of the endothelial permeability on the shear stresses, and on the LDL blood concentration. Sun et al. [7] have studied the influence of pulsatile flow on LDL transport and examine the validity of steady flow assumption. Yang and Vafai $[8,9]$ have developed a robust multi-layer porous model for the description of the mass transport in the arterial wall coupled with the mass transport in the arterial lumen. From their study, they have concluded that the LDL transport in the arterial wall is one-dimensional when a straight circular geometry is considered. Olgac et al. [10] have studied the effects of the local arterial wall shear stress distribution on the endothelial cell layer in order to accurately calculate LDL transport. They have chosen a realistic 3-D coronary artery segment with a single-layer porous arterial wall. Khakpour and Vafai [11] have critically assessed the different arterial transport models as far as the recent research activities on this issue are concerned with the help of the relevant governing equations in the study of fluid flow and mass transfer within the arteries by giving emphasis on the role of porous media.

From the available literature, it is noted that very little work has been done on the issue of quantifying the mass deposition of the plaque in the arterial wall. Therefore, in the present study, an attempt has been made to study initially the effect of fluid dynamic parameters on the disease, atherosclerosis and finally proposes a concept, from the idea of basic flow characteristics in constricted arteries, for the assessment of mass deposition in the arterial wall to some extent. The effect of Reynolds number and percentage of restriction on pressure drop through stenosis, velocity contour, velocity vector, and mass deposition in the wall is studied for the rectangular as well as half circular restrictions. Reynolds numbers are chosen as 100, 200, 300 and 400 and percentages of restrictions as $30 \%, 50 \%, 70 \%$ and $90 \%$ respectively.

\section{METHOD}

The governing Navier-Stokes and continuity equations are solved in the artery lumen with the commercial CFD code ANSYS 12.1. The pressure-velocity coupling equations are solved by SIMPLE (Semi-Implicit Method for Pressure-Linked Equations) algorithm. Least square cell based gradient, pressure: standard, momentum: first order momentum, are chosen for spatial discretization. The under relaxation factors for pressure as 0.3 , density as 1.0 and momentum as 0.7 are considered during simulation. The flow under consideration has been assumed to be steady, two-dimensional, laminar and axisymmetric, and fluid is considered to be Newtonian and incompressible. Uniform velocity at inlet, no slip condition at wall and zero pressure at exit are used in our simulation. Since coronary artery is an important artery for the disease of atherosclerosis, therefore the coronary artery (dia $=4 \mathrm{~mm}$ ) is taken for simulation. The density of blood and viscosity are considered as $1056 \mathrm{Kg} / \mathrm{m}^{3}$ and 0.0035 Pas respectively. The schematic diagrams of the computational domain selected for our study are illustrated in Figures 1(a) and (b) respectively. Total length of the computational domain is taken as $0.2 \mathrm{~m}$. Restriction is placed at the middle of the computational domain.

The results are generated for different Reynolds numbers of 100, 200, 300 and 400 and percentage of restriction of $30 \%, 50 \%, 70 \%$ and $90 \%$. The details of nodes and elements, considered during our study, are as follows in Table 1.

\section{RESULT AND DISCUSSIONS}

One of the most serious consequences of an arterial stenosis is the large pressure loss which may develop across a severe stenosis. The reduced pressure distal to the stenosis significantly alters the blood flow to the peripheral blood supplied by the artery. Atheromatuos plaques appear in the regions of low pressure because a suction action exerted on the surface endothelium eventually causes the layer to be selectively separated from adjacent tissue. This tearing action is thought to cause damage, in turn, to the endothelium and adjacent wall layers, with subsequent thickening of the intima and eventual plaque development [12]. The pressure loss is primarily dependent on the flow rate and the geometry of the stenosis due to relatively constant fluid properties of density and apparent viscosity. Moreover, the initiation and progression of atherosclerosis are dependent on the accumulation of LDL in the artery wall. One of the biomechanical forces of the chance of the deposition is depending on transmural flux. Moreover, the recirculation zone in the post stenotic region is considered to be an important phenomenon for fluid flux [13]. Since the fluid flux also depends on pressure of the blood, therefore, the pressure of blood at any section of the concerned artery and the pressure drop across the restricted zone may be thought to offer an idea to some extent regarding the formation and propagation of atherosclerosis. The physi- 


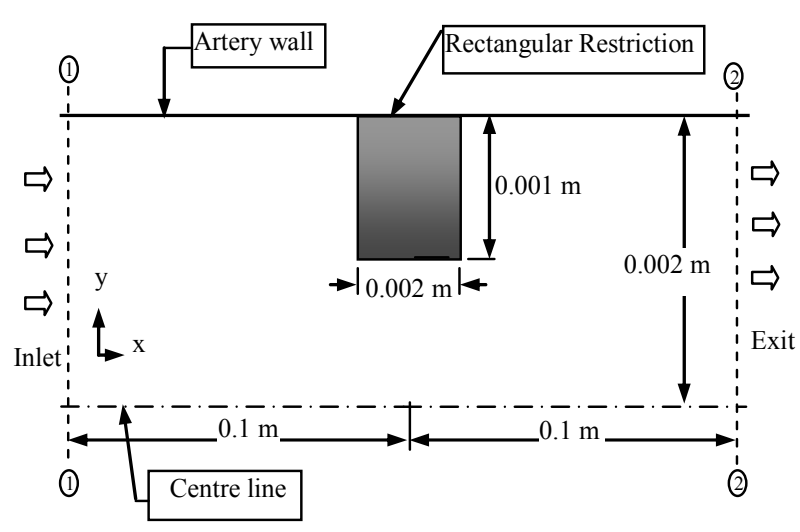

(a)

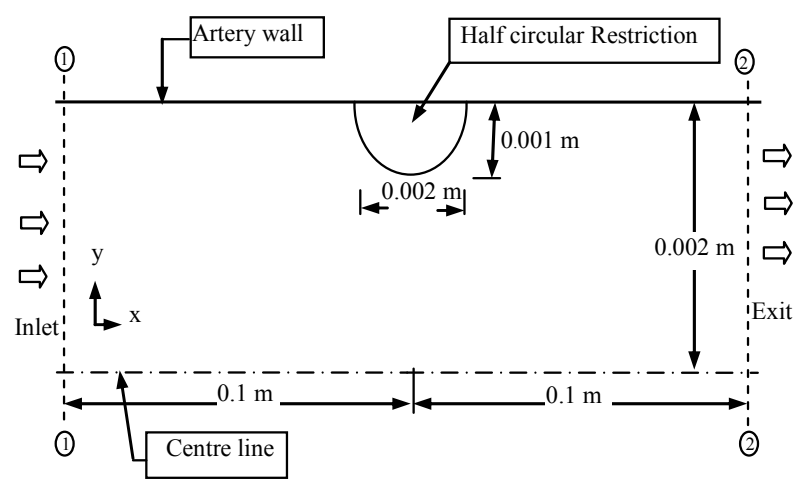

(b)

Figure 1. (a) Computational domain for $50 \%$ rectangular restriction. (b) Computational domain for $50 \%$ half circular restriction.

Table 1. Details of nodes and elements of the computational domain.

\begin{tabular}{ccccc}
\hline & $\mathrm{PR}=30 \%$ & $\mathrm{PR}=50 \%$ & $\mathrm{PR}=70 \%$ & $\mathrm{PR}=90 \%$ \\
\hline Nodes & 32,033 & 32,961 & 34,473 & 35,818 \\
Elements & 152,419 & 157,389 & 165,269 & 172,545 \\
\hline
\end{tabular}

ological significance of the recirculation zone is that the bloodstream stagnates locally in this area and allows platelets and fibrin to form a mesh at the inner wall in which lipid particles become trapped and eventually coalesce to form atheromatous plaque, this may tend to accumulate to cause a more severe stenosis [14]. After the recirculation zone, the blood reattaches on the arterial wall. This point of reattachment having the significance on the formation and propagation of atherosclerosis. The high cell turnover rate takes place near the reattachment point due to high cell division and cell density near that region. For this, a leaky junction may develop which is considered to be the possible pathway for transport of LDL through the arterial wall [15]. This phenomenon may be thought to assist the deposition. The recirculation zone can also be conceived with the use of velocity contour and velocity vectors. The quantification of mass deposition of macromolecules inside the arterial wall may be considered to be an important parameter in assessing the extent of disease, atherosclerosis. In the present numerical work, the effect of Reynolds number and percentage of restriction on pressure drop through stenosis, velocity contour, velocity vector, and mass deposition in the wall is studied for our considered rectangular as well as half circular restrictions. Reynolds numbers are chosen as 100, 200, 300 and 400 and percentage of restrictions as $30 \%, 50 \%, 70 \%$ and $90 \%$ respectively. In this work, the mass deposition is computed from the size of recirculation zone and finally the formulation, used for the computation is as, deposition $=2 / 3$ (stenosis height (stenosis length + reattachment length) $\pi$ Diameter of artery density of plaque material. Where $2 / 3$ is assumed to present the volume covered under the considered cases, and the magnitude of density of the deposited material is taken as $1.04 \mathrm{gm} / \mathrm{ml}$, which is the density of LDL.

Figure 2 shows the effect of Reynolds number and percentage of restriction on the pressure drop in the stenosis zone of a rectangular restriction. In case of Figure 2(a), the percentage of restriction has been fixed as $50 \%$. It is noted from the figure that as Reynolds number increases, the pressure drop across the stenosis increases. Figure 2(b) presents the variation of the pressure drop across the rectangular stenosis with percentage of restriction at fixed Reynolds number of 100 . From the figure, it is observed that initially upto $50 \%$, the increase in the pressure drop is less, then from $50 \%$ to $70 \%$, the pressure drop increases moderately, after that the pressure drop enhances drastically. Comparing Figures 2(a) and (b), it is also observed that the magnitude of pressure drop increase is more prominent in case of increase in the percentage of restriction compared to Reynolds number.

Figure 3 shows the variation of pressure drop with Reynolds number and percentage of restriction in case of half circular stenotic model. Here also for Figure 3(a), percentage of restriction has been considered to be $50 \%$, and for Figure 3(b), Reynolds number has been considered as 100. The nature of both the Figures 3(a) and (b) are more or less same as observed in case of Figures 2(a) and (b). In case of this model the magnitude of pressure drop for each case is observed to be significantly less than that of the corresponding each case of rectangular stenotic model. This is expected because the flow of fluid in poststenotic region is smoother in case of half circular stenosis model due to its favorable geometric feature than that of rectangular one.

From this study, it is concluded that the effect of percentage of restriction is more dominant than Reynolds number on the progression of the disease, atherosclerosis for any shaped restriction. Moreover, the rectangular shaped restriction is noted to be more prone to this disease. 


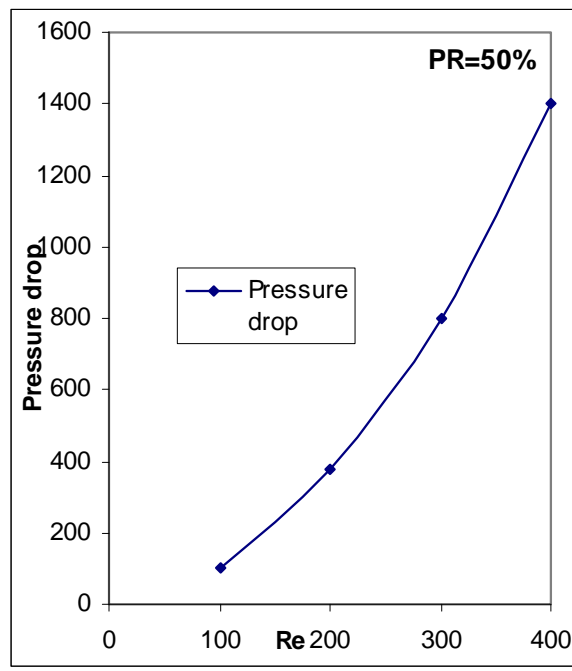

(a)

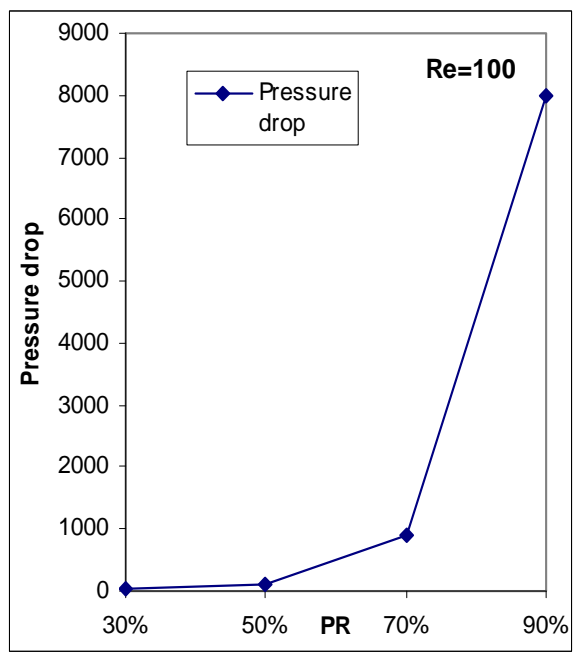

(b)

Figure 2. Effect of Re and PR on pressure drop for rectangular restriction.

Figure 4 represents the velocity contour and velocity vector for rectangular shaped restriction. From the figure, it is noted that the negative velocity zone increases with both Reynolds number and percentage of restriction. This indicates that with the increase in Reynolds number and percentage of restriction, there is more possibility of mass influx into arterial wall leading to higher mass deposition in the artery. Figure 5 shows the velocity contour and velocity vector for half circular shaped restriction. The nature of flow separation is similar as observed in case of rectangular stenotic model. Here the magnitude of recirculating zone at the post stenotic region is less.

Therefore from the Figures $\mathbf{4}$ and 5, it is revealed that the physiological effect of recirculation zone on the disease is less in case of half circular stenotic model. From the figures, it is expected that the magnitude of the mass

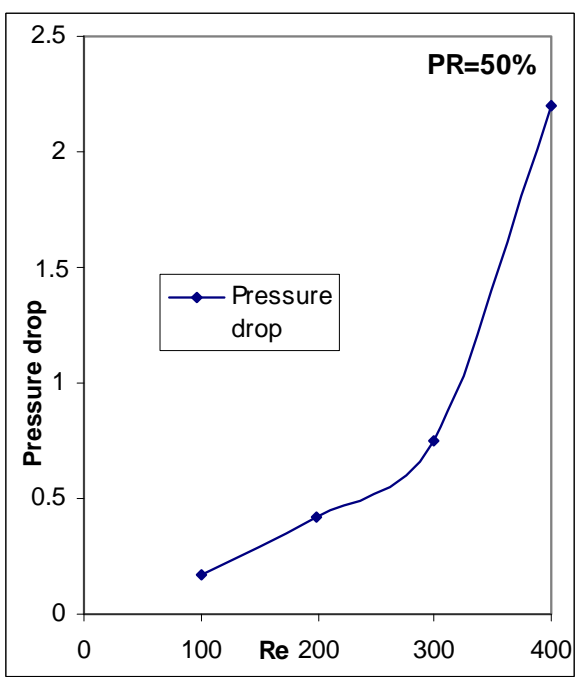

(a)

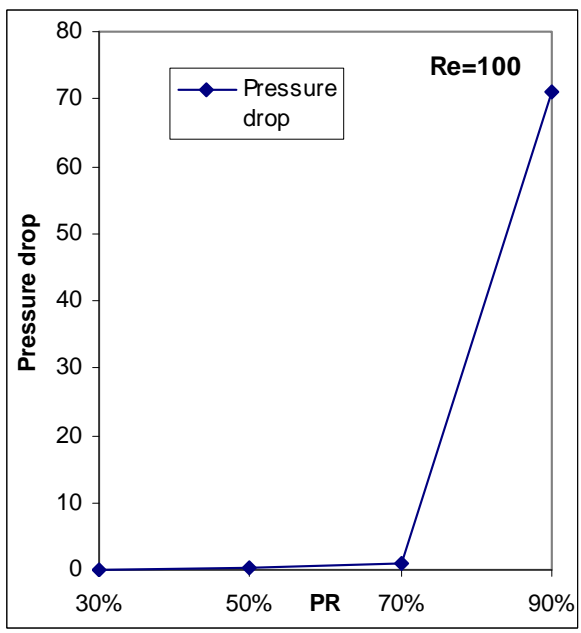

(b)

Figure 3. Effect of Re and PR on pressure drop for half circular restriction.

deposition will increase with the increase either Reynolds number or percentage of restriction for both the considered stenotic models. The chance of deposition is expected to be less for half circular stenotic model than that of rectangular one.

As per our expectation, we have observed that the magnitude of deposition in the artery wall increases with increase in Reynolds number or percentage of restriction in case of rectangular shaped restriction. This observation has been depicted in Figure 6. The effect of Reynolds number and the effect of percentage of restriction on mass deposition have been presented in Figures 6(a) and (b) respectively. The rate of increase in deposition with increase in percentage of restriction is observed to be more than that of increase in deposition rate with increase in Reynolds number for the said stenotic model. These results highlight that the effect of percentage of 


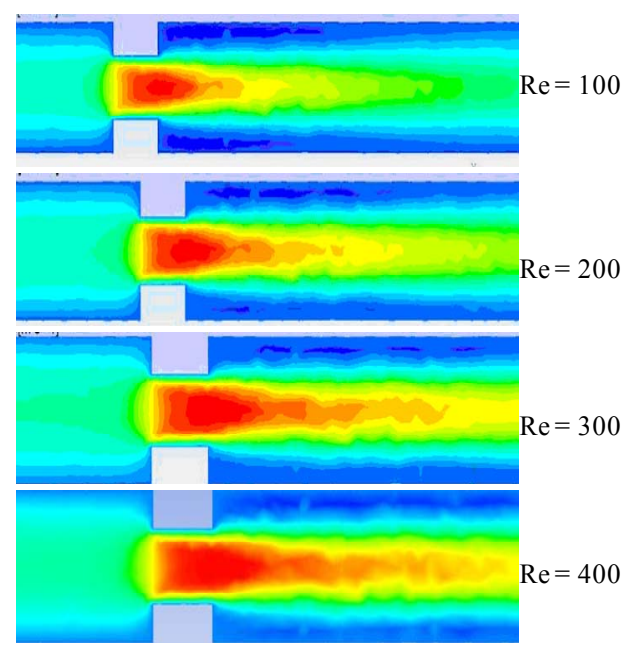

(a)

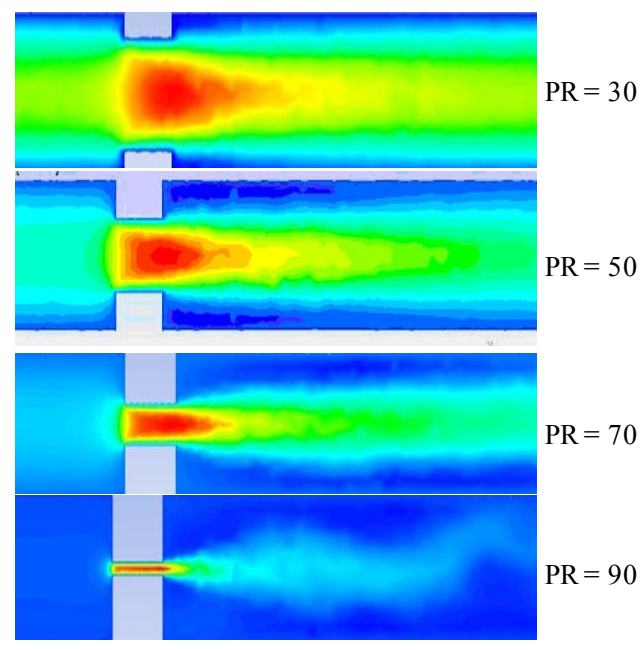

(c)

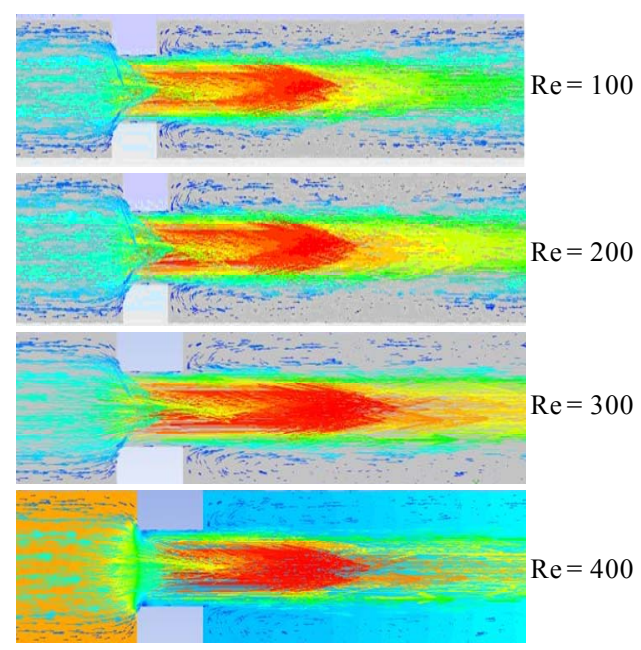

(b)

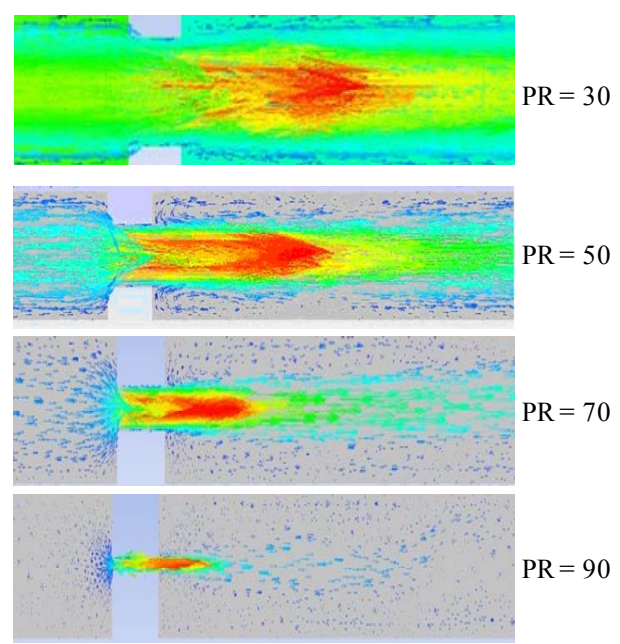

(d)

(a) Velocity contours at 50\% restriction. (b) Velocity vectors at 50\% restriction. (c) Velocity contours at $R e=100$. (d) Velocity vectors at $\mathrm{Re}=100$.

Figure 4. Effect of Re and PR on velocity contour and velocity vector for rectangular restriction.

restriction is more than that the effect of Reynolds number for the progression of atherosclerosis, which substantiates our earlier observations from pressure drop, velocity contour and velocity vector.

Figure 7 highlights the variation of deposition of the plaques in the arterial wall for the half circular stenotic model. Figure 7(a) represents the effect of Reynolds number on the said variation and Figure 7(b) represents the effect of percentage of restriction. From the figures, the similar nature of increasing trend of mass deposition is seen for this considered model except the magnitude of deposition compared to the case of rectangular shaped model.

Comparing the computed mass deposition results for rectangular and half circular stenotic models, it may be stated that the effect of percentage of restriction is more prone to the disease than that of Reynolds number, and half circular stenotic shape insists the less chance of mass deposition in the arterial wall.

\section{CONCLUSION}

In the present numerical work, the effect of Reynolds number and percentage of restriction on pressure drop through stenosis, velocity contour, velocity vector, and finally by proposing a concept, from the idea of basic flow characteristics in constricted arteries, for the assessment of mass deposition in the wall, and the effect of Reynolds number and percentage of restriction on mass deposition have been studied for our considered rectangular as well as half circular restrictions. Reynolds numbers are chosen as 100, 200, 300 and 400 and percentages of restrictions as $30 \%, 50 \%, 70 \%$ and $90 \%$ respectively. From this study of variation of pressure drop, it is also 


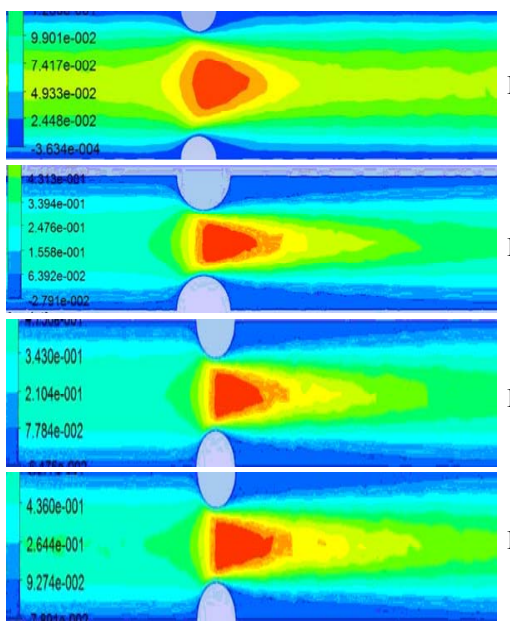

(a)

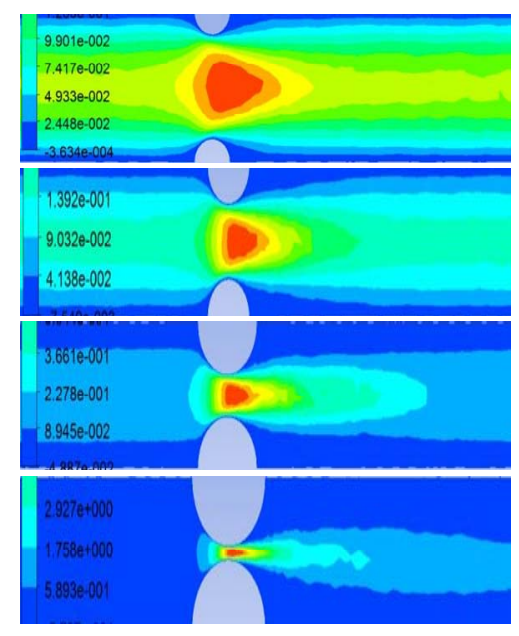

(c)

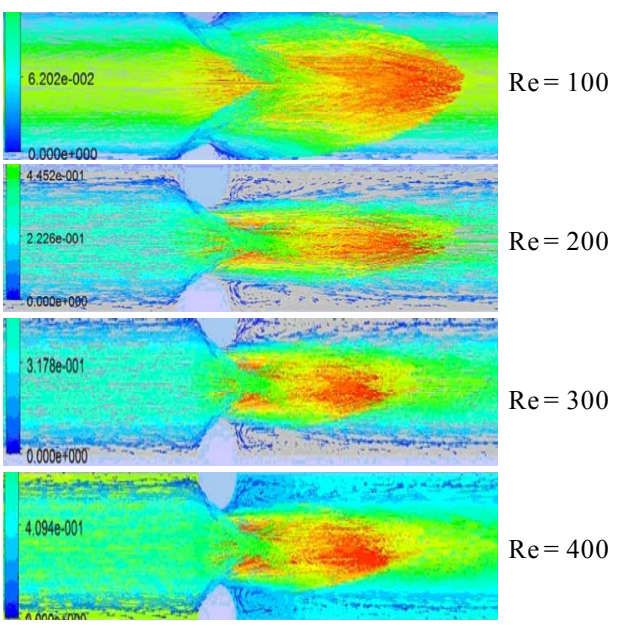

(b)

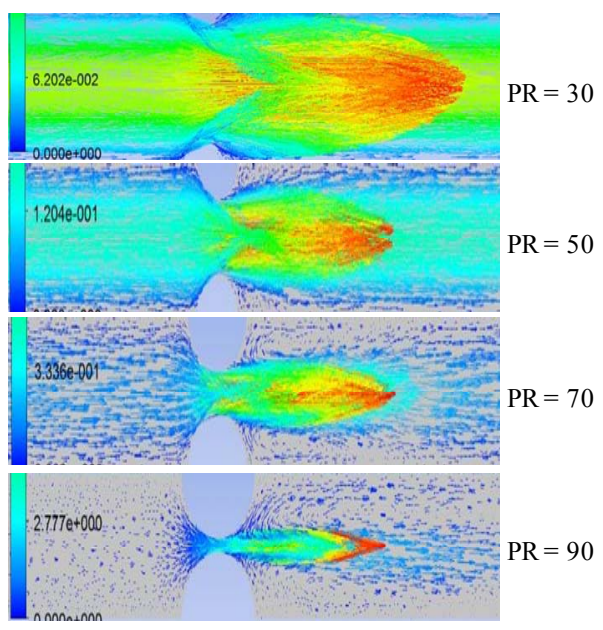

(d)

(a) Velocity contours at $50 \%$ restriction. (b) Velocity vectors at $50 \%$ restriction. (c) Velocity contours at $\mathrm{Re}=100$. (d) Velocity vectors at $R e=100$.

Figure 5. Effect of Re and PR on velocity contour and velocity vector for half circular restriction.

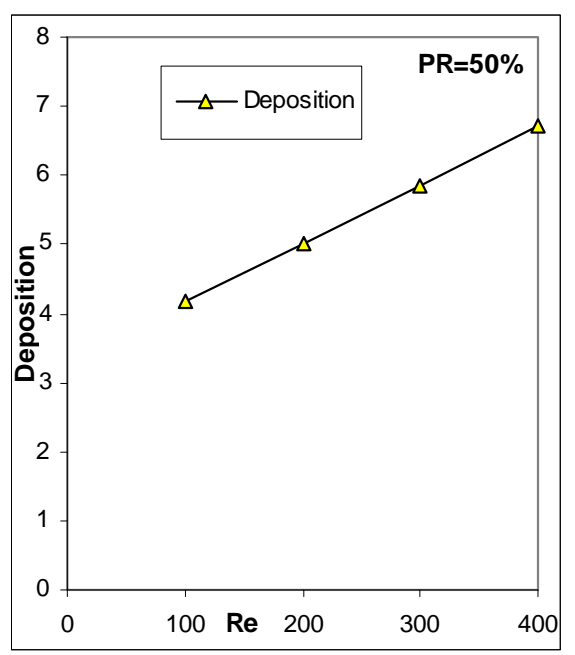

(a)

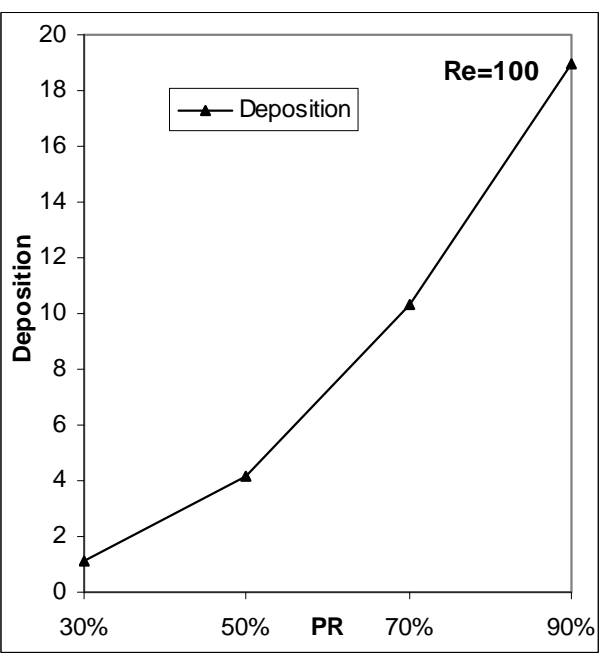

(b)

Figure 6. Effect of Re and PR on deposition for rectangular restriction. 


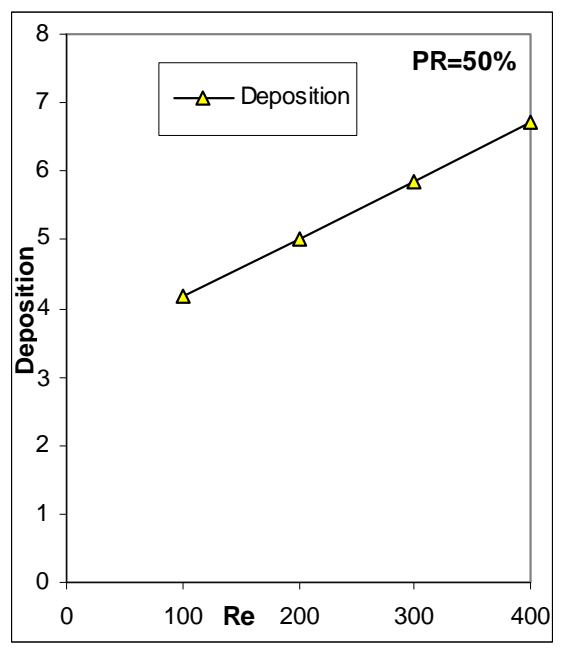

(a)

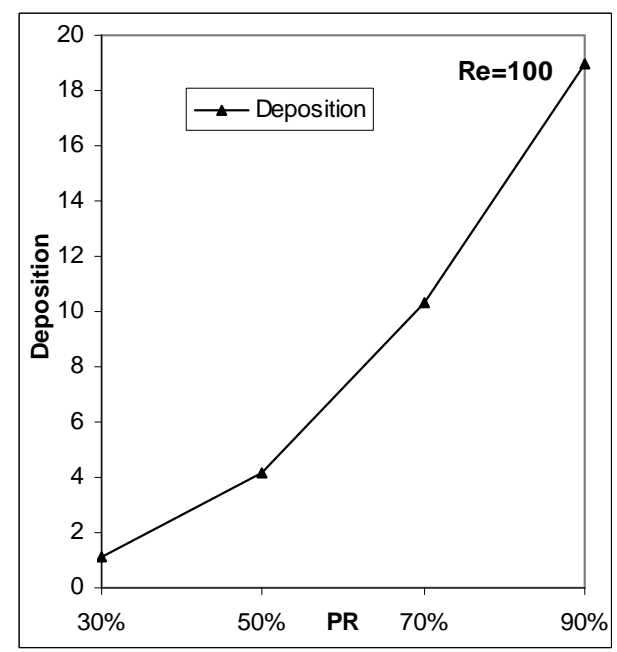

(b)

Figure 7. Effect of deposition on deposition Re and PR for half circular restriction.

revealed that the magnitude of pressure drop increase is more prominent in case of increase in the percentage of restriction compared to Reynolds number. The study of flow separation zone focuses on the effect of percentage of restriction that is more dominant than Reynolds number on the progression of the disease, atherosclerosis for any shaped restriction. Moreover, the rectangular shaped restriction is noted to be more prone to this disease. The mass deposition results of rectangular and half circular stenotic models motivate to conclude that the effect of percentage of restriction is more prone to the disease than that of Reynolds number, and half circular stenotic shape insists for the less chance of mass deposition in the arterial wall.

\section{ACKNOWLEDGEMENTS}

This work is supported by AICTE, RPS Scheme, (1023/POR/RID/RPS101/2009/10).

\section{REFERENCES}

[1] Stangeby, D. and Ethier, C. (2002) Computational analysis of coupled blood-wall arterial LDL transport. ASME Journal of Biomechanical Engineering, 124, 1-8. http://dx.doi.org/10.1115/1.1427041

[2] Kaazempur-Mofrad, M., Wada, S., Myers, J. and Ethier, C. (2005) Mass transport and fluid flow in stenotic arteries: Axisymmetric and asymmetric models. International Journal of Heat and Mass Transfer, 48, 4510-4517. http://dx.doi.org/10.1016/j.ijheatmasstransfer.2005.05.004

[3] Chakravarty, S. and Sen, S. (2006) A mathematical model of blood flow and convective diffusion, processes in constricted bifurcated arteries. Korea-Australia Rheology Journal, 18, 51-65.

[4] Sun, N.F., Torii, R., Wood, N.B., Hughes, A.D., Thom, S.A.M. and $\mathrm{Xu}, \mathrm{X} . Y$. (2006) Fluid-wall modelling of
LDL transport in a human right coronary artery. Excerpt from the Proceedings of the COMSOL Users Conference, Birmingham.

[5] Valencia, A. and Villanueva, M. (2006) Unsteady flow and mass transfer in models of stenotic arteries considering fluid-structure interaction. International Communications in Heat and Mass Transfer, 33, 966-975. http://dx.doi.org/10.1016/j.icheatmasstransfer.2006.05.006

[6] Gessaghia, V.C., Raschib, M.A., Larreteguym A.E. and Perazzo, C.A. (2007) Influence of arterial geometry on a model for growth rate of atheromas. Journal of Physics: Conference Series, 90, Article ID: 012046.

[7] Sun, N.F., Wood, N.B., Hughes, A.D., Thom, S.A.M. and $\mathrm{Xu}, \mathrm{X.Y}$. (2007) Influence of pulsatile flow on LDL transport in the arterial wall. Annals of Biomedical Engineering, 35, 1782-1790. http://dx.doi.org/10.1007/s10439-007-9347-1

[8] Yang, N. and Vafai, K. (2008) Low-density lipoprotein (LDL) transport in an artery-A simplified analytical solution. International Journal of Heat and Mass Transfer, 51, 497-505. http://dx.doi.org/10.1016/j.ijheatmasstransfer.2007.05.023

[9] Yang, N. and Vafai, K. (2006) Modeling of low-density lipoprotein (LDL) transport in the artery-Effects of hypretension. International Journal of Heat and Mass Transfer, 49, 850-867.

http://dx.doi.org/10.1016/j.ijheatmasstransfer.2005.09.019

[10] Olgac, U., Kurtcuoglu, V. and Poulikakos, D. (2008) Modeling of blood-wall low-density lipoprotein mass transport in dependence of shear stress. Journal of Biomechanics, 41, S277. http://dx.doi.org/10.1016/S0021-9290(08)70276-8

[11] Khakpour, M. and Vafai, K. (2008) Critical assessment of arterial transport models. International. Journal of Heat and Mass Transfer, 51, 807-822.

http://dx.doi.org/10.1016/j.ijheatmasstransfer.2007.04.021

[12] Gessner, F.B. (1973) Haemodynamic theories of atehrogenesis. Circulation Research, 3, 259-266. 
http://dx.doi.org/10.1161/01.RES.33.3.259

[13] Chesler, N.C. and Enyinna, O.C. (2003) Particle deposition in arteries ex vivo: Effects of pressure, flow and wave form. Journal of Biomechanical Engineering, 125, 389394. http://dx.doi.org/10.1115/1.1572905

[14] Tu, C., Deville, M., Dheur, L. and Vanderschuren, L., (1992) Finite element simulation of pulsatile flow

\section{NOMENCLATURES}
A: Area at any section, $\left[\mathrm{m}^{2}\right]$
D: Dia of the artery, $[\mathrm{m}]$
R: Radius of the artery, $[\mathrm{m}]$
L: Total length of computational domain, [m]
p: Static pressure, $\left[\mathrm{Nm}^{-2}\right]$

through arterial stenosis. Journal of Biomechanics, 25, 1141-1152.

http://dx.doi.org/10.1016/0021-9290(92)90070-H

[15] Weinbaum, S. and Chien, S., (1993) Lipid transport aspects of atherogenesis. Journal of Biomechanical Engineering, 115, 602-610.

http://dx.doi.org/10.1115/1.2895547

Re: Reynolds number

$\mathrm{U}$ : Average velocity in r-direction at inlet, $\left[\mathrm{ms}^{-1}\right]$

r: Density, $\left[\mathrm{kg} \cdot \mathrm{m}^{-3}\right]$

$\mu$ : Dynamic viscosity, [Pas]

PR: Percentage of restriction (by diameter) 